Old Dominion University

ODU Digital Commons

CCPO Publications

Center for Coastal Physical Oceanography

1995

\title{
Growth Characteristics Downstream of a Shallow Bump: Computation and Experiment
}

Ronald D. Joslin

Chester E. Grosch

Old Dominion University, cxgrosch@odu.edu

Follow this and additional works at: https://digitalcommons.odu.edu/ccpo_pubs

Part of the Fluid Dynamics Commons, and the Oceanography Commons

\section{Repository Citation}

Joslin, Ronald D. and Grosch, Chester E., "Growth Characteristics Downstream of a Shallow Bump: Computation and Experiment" (1995). CCPO Publications. 186.

https://digitalcommons.odu.edu/ccpo_pubs/186

\section{Original Publication Citation}

Joslin, R.D., \& Grosch, C.E. (1995). Growth-characteristics downstream of a shallow bump: Computation and experiment. Physics of Fluids, 7(12), 3042-3047. doi: 10.1063/1.868680

This Article is brought to you for free and open access by the Center for Coastal Physical Oceanography at ODU Digital Commons. It has been accepted for inclusion in CCPO Publications by an authorized administrator of ODU Digital Commons. For more information, please contact digitalcommons@odu.edu. 


\title{
Growth characteristics downstream of a shallow bump: Computation and experiment
}

\author{
Ronald D. Joslin \\ NASA Langley Research Center, Hampton, Virginia 23681-0001 \\ Chester E. Grosch \\ Old Dominion University, Norfolk, Virginia 23529
}

(Received 1 March 1995; accepted 24 July 1995)

\begin{abstract}
Measurements of the velocity field created by a shallow bump on a wall revealed that an energy peak in the spanwise spectrum associated with the driver decays and an initially small-amplitude secondary mode rapidly grows with distance downstream of the bump. Linear theories could not provide an explanation for this growing mode. The present Navier-Stokes simulation replicates and confirms the experimental results. Insight into the structure of the flow was obtained from a study of the results of the calculations and is presented. (c) 1995 American Institute of Physics.
\end{abstract}

\section{INTRODUCTION}

In order to determine whether an eigenmode of the linearized boundary-layer cquations or an Orr-Sommerfeld approach was appropriate for studying the disturbance field created by a localized boundary perturbation of houndary-layer flow, Gaster, Grosch, and Jackson ${ }^{1}$ performed an experiment to find out what the solution should look like. Surprisingly, the experimental results did not seem to be consistent with either model. A significant result of the experiment was that a primary spanwise mode associated with the diaphragm dimension decayed slightly with downstream distance and a small secondary mode rapidly grew with downstream distance. To rule out any possible anomaly in the experiment, the present computational study was initiated to duplicate the experimental results. Equally important, the results of the computation permit a detailed study of the flow field structure and yield insight into the physics of the flow. The computations involve solving the unsteady nonlinear NavierStokes equations for a spatially growing boundary-layer flow and should be equivalent to an ideal experimental study.

\section{OVERVIEW OF EXPERIMENTAL CONDITIONS}

Although a detailed description of the experimental conditions was given by Gaster et al., ${ }^{\prime}$ a synopsis of the experimental parameters important for the computations is provided here. In the experiment, the bump was located $400 \mathrm{~mm}$ from the leading edge of the tlat plate. At this location, the boundary and displacement thicknesses of the undisturbed flow near the bump werc $\delta=2.88 \mathrm{~mm}$ and $\delta^{*}=0.99 \mathrm{~mm}$, respectively. The associated boundary-layer Reynolds numbers near the bump were $R_{\delta}=3480$ and $R_{\delta^{*}}=1196$. A silicon rubber diaphragm of $20 \mathrm{~mm}\left(\simeq 20 \delta^{*}\right)$ was used to force the disturbance. Most of the diaphragm motion occurred over $10-15 \mathrm{~mm}$ of the center. The amplitude of the bump motion was about $0.1 \mathrm{~mm}(100 \mu \mathrm{m})$, which is a typical height of the roughness element used in receptivity experiments (see Saric, ${ }^{2}$ Sec. 3.1.1). A measure of the disturbance amplitude near the bump was predicted to be about $u=4.9 \%$ of the free-stream velocity. Although a stationary bump would be preferable, a forcing frequency of $2 \mathrm{~Hz}$ was used to discrimi- nate the signal created by the bump from the background noise present to some degree in all experiments. There are, of course, Tollmien-Schlichting modes with a $2 \mathrm{~Hz}$ frequency but these are highly damped at the Reynolds numbers of the experiment. The $2 \mathrm{~Hz}$ frequency is well below that of any growing Tollmien-Schlichting mode. The $4.9 \%$ disturbance amplitude is too large to enable a comparison of the experimental disturbance with receptivity theory, which, to date, is based on the infinitesimal small-amplitude assumption. It is possible that a sufficiently large disturbance at very low frequency or even a steady disturbance could cause a bypass transition, but no evidence of transition or turbulence was observed in the experiment or in the computations reported here. Measurement stations were set up about 70 and 105 boundary-layer thicknesses downstream of the bump, $(\approx 200$ and 300 displacement thicknesses). These are station $B$, section $\mathrm{b}$ b and station $\Lambda$, section $\mathrm{c}-\mathrm{c}$, respectively, as shown in Fig. 1 of Gaster et al. ${ }^{1}$ The Reynolds numbers at these measuring stations were approximately $R_{\delta^{*}}=1466$ and $R_{\delta^{*}}=1585$. Detailed measurements of the streamwise component profiles in both the normal and spanwise directions were made at these stations. Less detailed measurements were made over a larger area.

\section{NUMERICAL METHOD OF SOLUTION}

The numerical techniques required for the simulation and the disturbance forcing are briefly discussed in this section. For a detailed description of the spatial DNS (NavierStokes) approach used for this study, refer to Joslin, Streett, and Chang. ${ }^{3.4}$ The instantaneous velocities $\tilde{\mathbf{u}}=(\tilde{u}, \tilde{v}, \tilde{w})$ and the pressure $\tilde{p}$ are decomposed into steady base and disturbance components. The base flow is given by velocities $\mathbf{U}=(U, V, W)$ and the pressure $P$; the disturbance is given by velocities $\mathbf{u}=(u, v, w)$ and the pressure $p$. The velocities correspond to the coordinate system $\mathbf{x}=(x, y, z)$, where $x$ is the streamwise direction, $y$ is the wall-normal direction, and $z$ is the spanwise direction. The base flow for the flat plate can be reasonably approximated by the Blasius similarity solution $\mathbf{U}=(U, V, 0)$, and the disturbance flow is found by solving the three-dimensional, incompressible Navier-Stokes equations. These equations are the momentum equations, 


$$
\mathbf{u}_{\mathbf{t}}+(\mathbf{u} \cdot \boldsymbol{\nabla}) \mathbf{u}+(\mathbf{U} \cdot \boldsymbol{\nabla}) \mathbf{u}+(\mathbf{u} \cdot \boldsymbol{\nabla}) \mathbf{U}=-\nabla p+\frac{1}{R} \nabla^{2} \mathbf{u},
$$

and the continuity equation,

$$
\nabla \cdot \mathbf{u}=0 \text {. }
$$

The boundary conditions in the farfield are

$$
\mathbf{u} \rightarrow 0, \quad \text { as } y \rightarrow \infty,
$$

and the conditions at the wall are

$$
\mathbf{u}=\mathbf{u}_{\mathrm{c}}, \quad \text { at } \mathbf{y}=0 \text {, }
$$

where $\mathbf{u}_{c}=0$, except for the portion of the wall that models the bump. The Reynolds number $R=U_{\infty} \delta_{0}^{*} / \nu$ is based on the boundary-layer displacement thickness at the inflow of the computational domain, the free-stream velocity $U_{x}$, and the kinematic viscosity $\nu$.

To solve Eqs. (1)-(3), computationally, the spatial discretization entails a Chebyshev collocation grid in the wallnormal direction, fourth-order finite differences for the pressure equation, sixth-order compact differences for the momentum equations in the streamwise direction, and a Fourier sine and cosine series in the spanwise direction on a staggered grid. ${ }^{4}$ For time marching, a time-splitting procedure is used with implicit Crank-Nicolson differencing for normal diffusion terms and an explicit three-stage RungeKutta method. ${ }^{5}$ The influence-matrix technique is employed to solve the resulting pressure equation (HelmholtzNeumann problem) ${ }^{6.7}$ At the inflow boundary, the mean base flow is forced and, at the outflow, the buffer-domain technique of Streett and Macaraeg ${ }^{8}$ is used.

The boundary conditions that have to be imposed to represent an oscillating bump are a streamwise velocity perturbation related to the mean shear and the bump height, together with the normal velocity of the bump, as described in Gaster et al. ${ }^{\prime}$ Although the experiments had to use a lowfrequency oscillating bump, the computations can use a stationary bump. Hence, the boundary conditions reduce to

$$
u(x, 0, z)=-h(x, z) \frac{d U}{d y} .
$$

The form $h(x, z)=v_{x} \sin (x)^{3} \sin (z)^{3}$ is imposed for the bump shape, which yields a computationally smooth bump. The bump height is given by the amplitude $v_{w}=10 \%$. Although no attempt was made to exactly match the ingested disturbance amplitude of $4.9 \%$ in the experiment, an amplitude of approximately $3.4 \%$ was observed in the computations. The streamwise length of the bump was $15.9 \delta_{0}^{*}$ and the spanwise half-length was $6.5 \delta_{0}^{*}$. As will be seen in the results section of this paper, the somewhat arbitrary selection of the amplitude and shape of the computational bump did not have an adverse effect on the desired comparison with experiments.

Figure 1 is a sketch of the computational domain. In the experiment the displacement thickness at the bump, $\delta_{0}^{*}$ was approximately $1 \mathrm{~mm}$ and the boundary-layer Reynolds number at that location, $R=U_{x} \delta_{0}^{*} / \nu$, was 1200 . We choose $\delta_{0}^{*}$ as the length scale for the computation and set the Reynolds number in Eq. (1) to be 1200. As shown in Fig. 1, the farfield

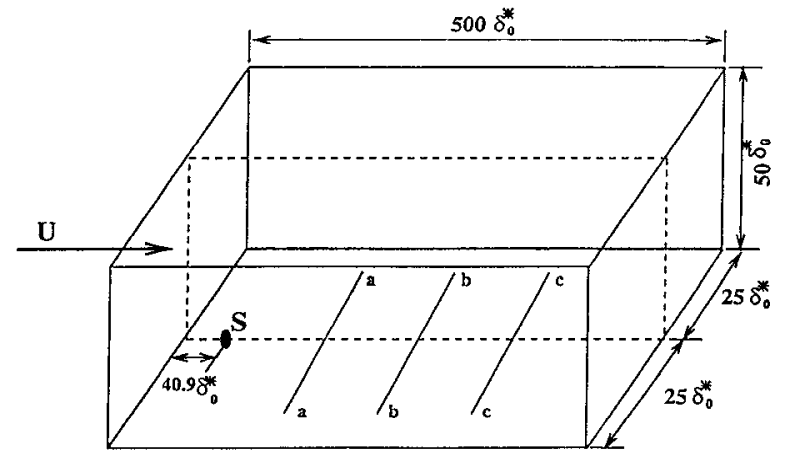

FIG. I. Sketch of the computational domain showing its size and the location of the bump. The lines $a-a, b-b$, and $c-c$ show the location of the similarly labeled lines of Gaster et al., along which measurements were made. We present results of the simulation on planes including lines $a$ - $a$ and $c-c$.

boundary was located $50 \delta_{0}^{*}$ from the wall, the streamwise extent of the domain was $500 \delta_{0}^{*}$ from the inflow, and the spanwise extent of the domain was $25 \delta_{0}^{*}$. This spanwise extent is shown by the dotted line in Fig. 1. Along this surface a symmetry condition on the flow field was applied. Thus the effective spanwise extent of the computational domain was $50 \delta_{0}^{*}$, as shown in Fig. 1. The center of the bump was positioned on the symmetry boundary at $40.9 \delta_{0}^{*}$ from the inflow, again as shown in Fig. 1. With the computational scale chosen to be $\delta_{0}^{*}=1 \mathrm{~mm}$, all of the dimensions shown in Fig. 1 of Gaster et al. ${ }^{1}$ can be directly translated into $\delta_{0}^{*}$ units. The lines labeled $a-a, b-b$, and $c-c$ in Fig. 1 are the similarly labeled lines shown in Fig. 1 of Gaster et al. ${ }^{1}$

The choice of grid, computational domain size, and time-step size were based on previous experience described in Joslin, Streett, and Chang ${ }^{3,4}$ for unsteady disturbances and in Joslin and Street ${ }^{9}$ for a stationary disturbance. The simulation used a coarse grid of 661 streamwise, 61 wall-normal, and 20 spanwise grid points (spanwise symmetric). For the time marching, a time-step size of 0.2 is chosen for the threestage Runge-Kutta method. The coarse grid computation required 44 Cray $2 \mathrm{~h}$ with a single processor to converge to a time-independent solution. In addition to the coarse grid calculation, a grid refinement simulation was performed to verify the quantitative accuracy of the results of the course grid computations reported below. This second simulation was conducted with a grid of 1321 streamwise, 81 wallnormal, and 39 spanwise grid points. This translates into doubling the grid in the streamwise and spanwise directions; in the wall-normal direction, Chebyshev series are used, which have coefficients that converge exponentially.

Because the disturbance excitation is steady and the resulting disturbance modes are stationary, the fine-grid simulation had initial conditions that correspond to the course grid final results. If the course and fine-grid results were time independent and quantitatively similar, then significant computational savings (approximately 300 Cray Y/MP hours) can be realized with this choice of initial conditions for the fine-grid simulation. The fine-grid simulation was marched in time and the results were compared after 180 and 420 time steps. The results were identical, indicating that the fine-grid 


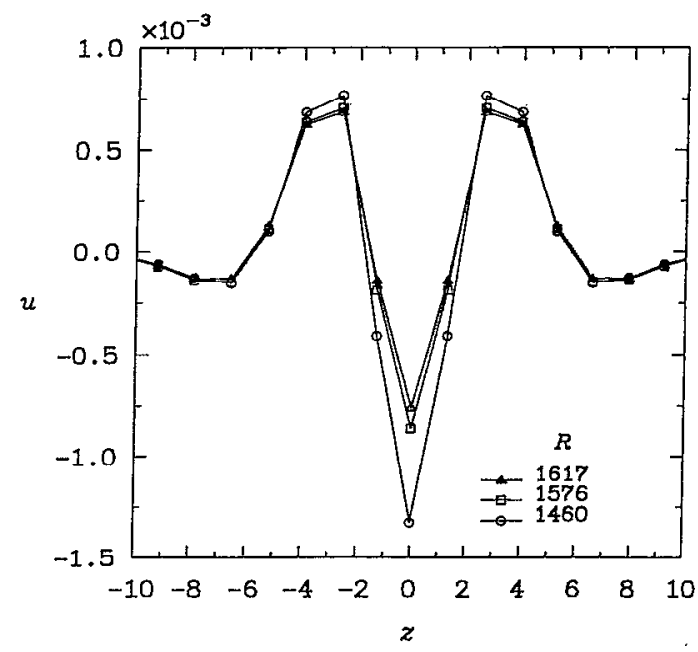

FIG. 2. Streamwise velocity profiles in the streamwise/spanwise plane downstream of the bump. The profiles are at a height of $y=\delta_{0}^{*}$.

simulation had converged after only 180 time steps. The cost of this simulation was 19 Cray Y/MP hrs. As will be shown below in Sec. IV, the results of the coarse and fine grid computations were essentially identical.

\section{RESULTS}

The results shown in Fig. 2 are spanwise profiles of the streamwise velocity component in the spanwise direction at $y \simeq \delta_{0}^{*}$, which is approximately the distance from the wall used in the experiments. This top view would have the bump placed at the bottom of the figure, and the flow direction is from bottom to top. As expected, there is a velocity deficit directly downstream of the bump and lobes of enhanced velocity on both sides of the bump. Note that the intensity of this deficit and lobes is decreasing with distance downstream of the bump. This qualitative picture matches the experimental observation, except there was some asymmetry in the experiments.

Figure 3(a) shows the variation with downstream distance of the total energy of the disturbance generated by the bump, as obtained from the fine- and coarse-grid simulations. Clearly, quantitative agreement is observed (note that the ordinate has a logarithmic scale). Figure 3(b) shows the variation of the total energy and the square of the velocity components with downstream distance. The total energy is decreasing with distance downstream and the streamwise velocity component is clearly dominant compared with the insignificant wall-normal and spanwise components. In the experiments, only the streamwise velocity component was recorded and the discussion and conclusions of the flow were described based on the streamwise velocity. The computations clearly show that it is unnecessary to consider the wall-normal and spanwise velocities.

Figure 4 shows the low-wave number modal decomposition of the streamwise velocity component in the spanwise direction. Confirming the experiments, the low-wave-number modes are growing with downstream distance; all other highwave-number modes $(\geqslant 4)$ are decaying everywhere. The

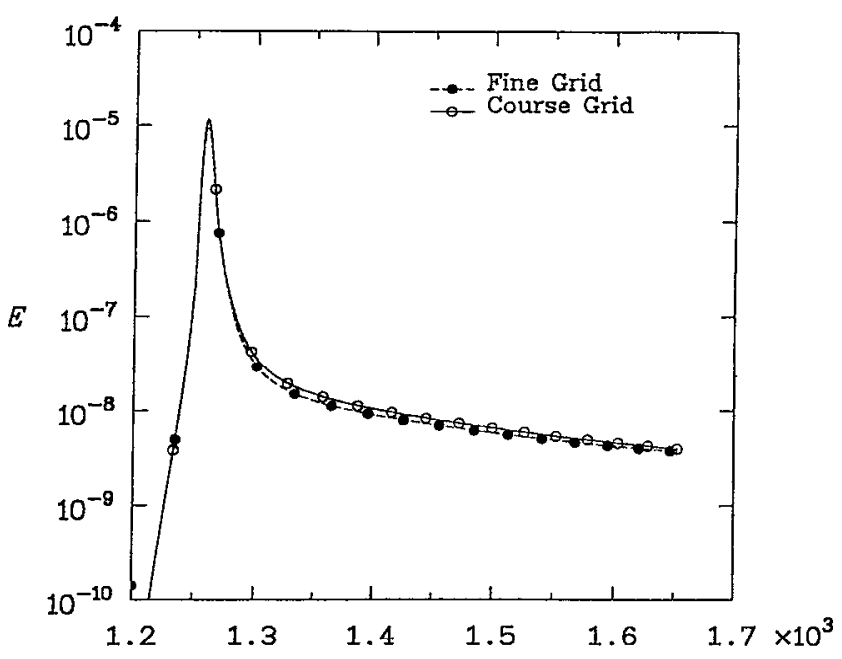

(a)

$R$

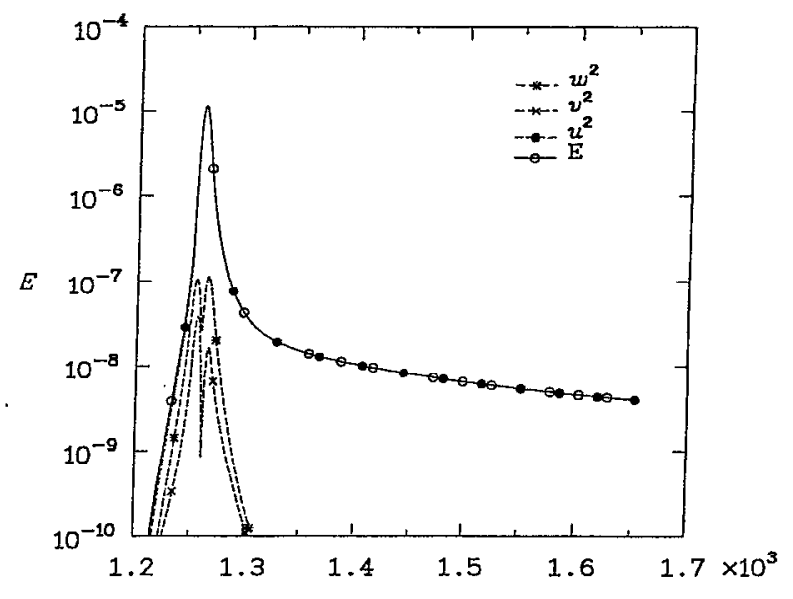

(b)

$R$

FIG. 3. (a) Variation of the total disturbance energy with downstream distance as obtained from the coarse- and fine-grid computations. (b) Variation with downstream distance of the total disturbance energy and that of the energy in each velocity component

low-wave-number modes are marked by a region of rapid growth followed by either an asymptote or decay region beyond the present computational domain. A growth in the $\beta=2$ mode by a factor of 8 in magnitude was noted by Gaster et al. ${ }^{1}$ Here, the dominant $\beta=2$ mode has grown by over a factor of 6 in magnitude and has not reached its maximum value within the computational domain.

The $\beta=2$ velocity profiles, obtained from both the coarse- and fine-grid simulations, are shown with distance from the wall in Fig. 5 at various downstream distances. The results of both simulations are in excellent quantitative agreement and both simulations show modal growth consistent with the experiments. The profiles at $R=1576$ and 1617 qualitatively match the experiments in shape and have their peak near $y \simeq 2 \delta^{*}$, as do the experimental results (see Fig. 3 in Gaster et al. ${ }^{1}$ ). The magnitudes are, however, different. The results of the calculations shown in Fig. 5 have a peak value of about $5 \times 10^{-5}$, while the measurements show a peak value of $2 \times 10^{-3}$. 


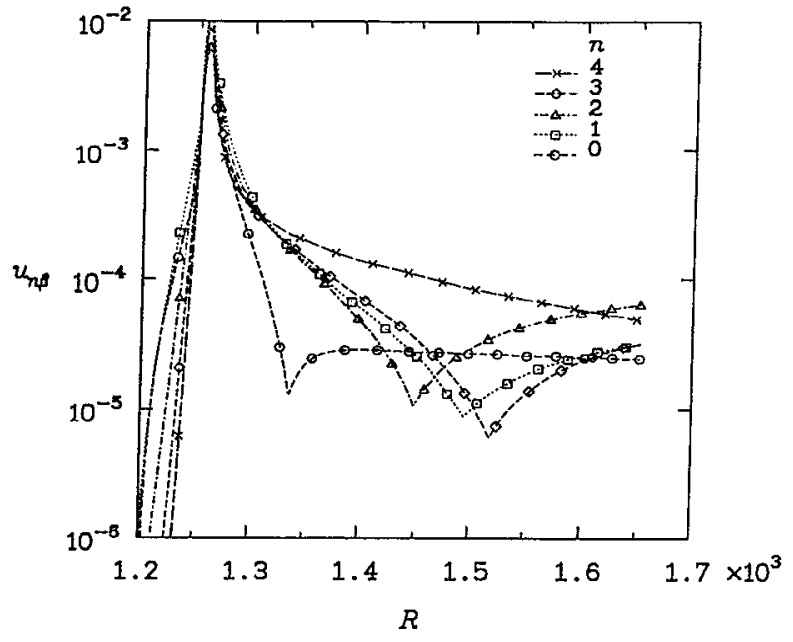

FIG. 4. Variation with downstream distance of the streamwise velocity component decomposed into spanwise modes.

The three-dimensional structure of the flow field can be inferred from the results presented in Figs. 6, 7, and 8. All of these results are on the $y-z$ plane at $x=503$, which is slightly downstream of the section a-a as shown in Fig. 1 of Gaster et al. ${ }^{1}$ and of this paper. At this location $R_{\delta^{*}} \approx 1388$. The results of the calculation are obtained on a Chebyshev collocation grid in the wall-normal $(y)$ direction. In the spanwise direction the computational results from $z=0$ to $z=25 \delta^{*}$ (with a symmetry boundary condition at $z=0$ ) were "folded" about $z=0$ in order to obtain the flow field in $-25 \delta^{*} \leqslant z \leqslant 25 \delta^{*}$. Although the farfield boundary is located at $y=50 \delta^{*}$, results are shown in $0 \leqslant y \leqslant 5 \delta^{*}$ because the disturbance field is essentially confined to the boundary layer. Because it is more convenient in presenting the data, the computational results were interpolated onto a uniform grid

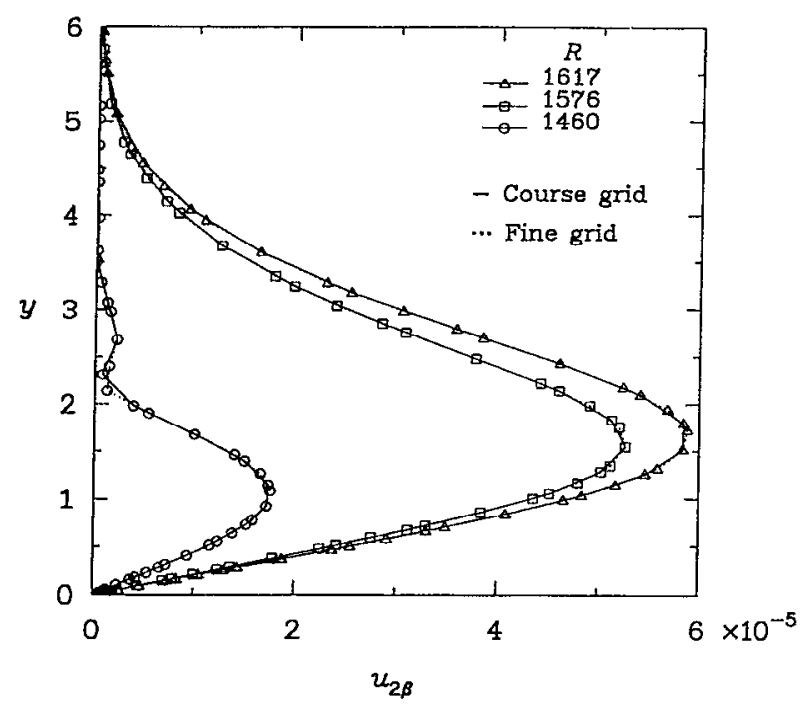

FIG. 5. The streamwise velocity component at three different downstream locations. The solid curves are the result of the coarsc-grid simulation and the dashed curves are the result of the fine-grid simulation. These curves coincide almost everywhere in this figure.

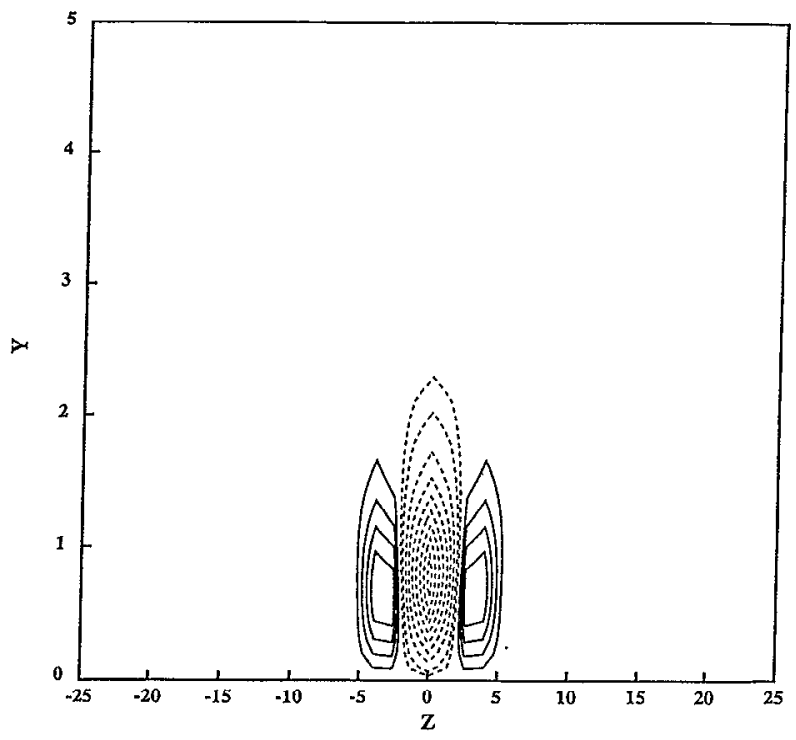

FIG. 6. Contours of $u$, the streamwise component of the disturbance velocity, on the $y-z$ plane at $x=503$. Contours with positive values of $u$ are solid and those with negative values are dashed. The contours values are $-1.6 \times 10^{-3}$ to $-0.2 \times 10^{-3}$ in steps of $0.2 \times 10^{-3}$ and from $-0.1 \times 10^{-3}$ to $0.5 \times 10^{-3}$ in steps of $0.1 \times 10^{-3}$, excluding 0.0 . The minimum value of $u$, $-1.69 \times 10^{-3}$, occurs on the centerline at $y=0.73$, with the maxima, $0.48 \times 10^{-3}$, being located at $y=0.73$ and $z= \pm 3.95$.

in the $y$ direction. It should be noted that Figs. 6, 7 and 8 are distorted by an, approximately, 10 to 1 stretching in the $y$ direction as compared to the $z$ direction.

Figure 6 contains contours of $u$, the streamwise component of the velocity. In this plane, $-1.69 \times 10^{-3}$ $\leqslant u \leqslant 0.48 \times 10^{-3}$ and the minimum occurs on the centerline at $y=0.73$, with the maxima being located at $y=0.73$ and $z= \pm 3.95$. This is the same structure seen in the profiles of $u$ at $y=\delta^{*}$ shown in Fig. 2. As seen from Fig. 6, the streamwise component of the disturbance field is an up-

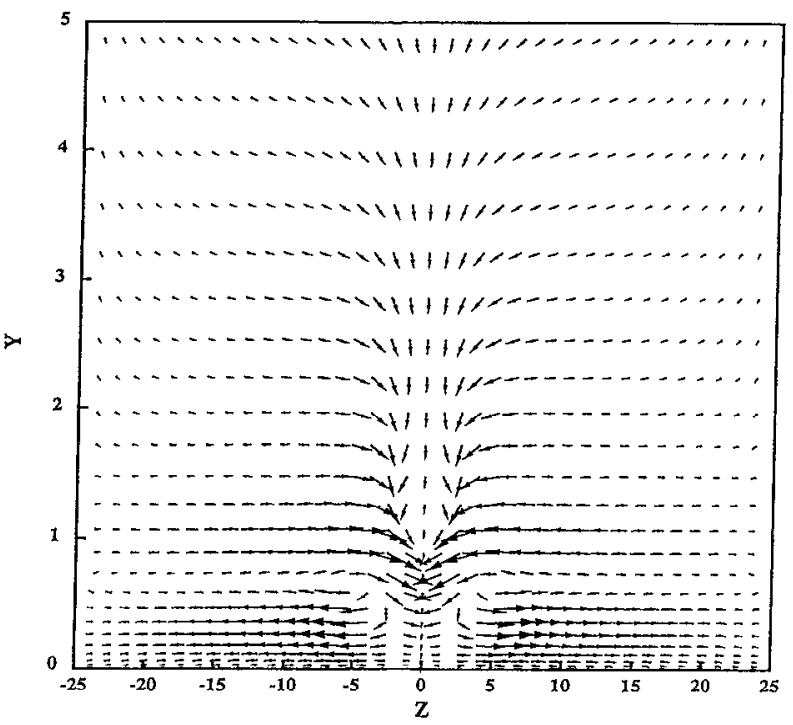

FIG. 7. Vectors of $(v, w)$ on the $y-z$ plane at $x-503$. The maxima of $\sqrt{v^{2}+w^{2}}$ are located at $y=0.35$ and $z= \pm 5.26$ and have the value $2.62 \times 10^{-5}$. 


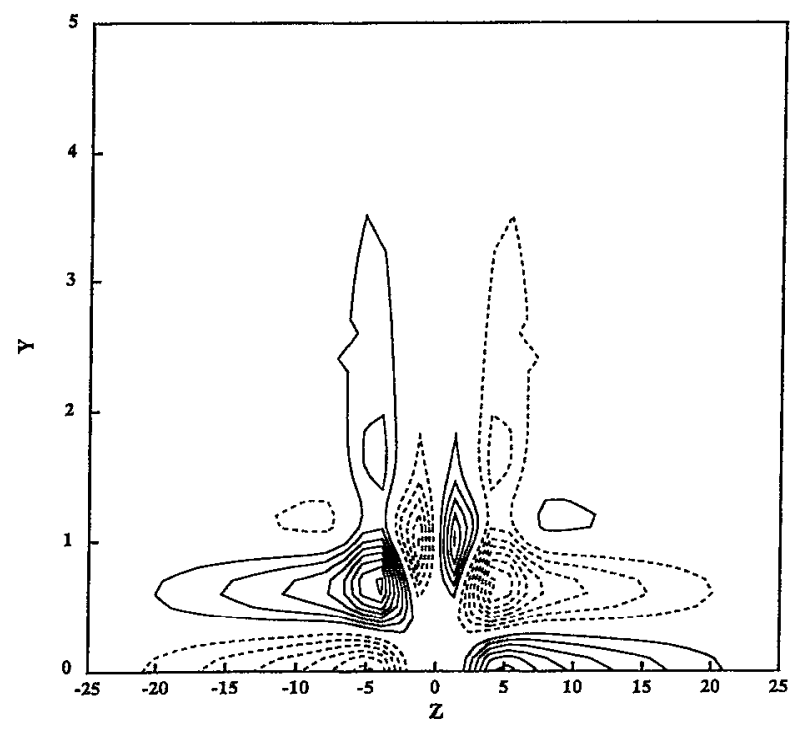

FIG. 8. Contours of $\omega_{x}$, the streamwise component of the disturbance vorticity, on the $y-z$ plane at $x=503$. Contours with positive values of $\omega_{x}$ are solid and those with negative values are dashed. Positive $\omega_{x}$ indicates clockwise rotation and negative counterclockwise. The contours values are $-1.6 \times 10^{-4}$ to $1.6 \times 10^{-4}$ in steps of $0.2 \times 10^{-4}$, excluding 0.0 . The minimum value of $\omega_{x},-1.64 \times 10^{-4}$, is at $y=0.70$, and $z=3.95$, and the maximum, $1.64 \times 10^{-4}$, is at $y=0.70$ and $z=-3.95$.

stream flowing "jet" on the centerline with downstream counterflowing jets on both sides. The entire field is essentially confined to the inner part of the boundary laycr $(y \leqslant 2)$.

The $(v, w)$ vectors in the same $x$ plane are shown in Fig. 7. The maximum of $\sqrt{v^{2}+w^{2}}$ is $2.62 \times 10^{-5}$ and occurs at $y=0.35$ and $z= \pm 5.26$. There is an inflow toward the region of the upstream "jet" along the centerline and an outflow between the downstream "jets" and the wall. Both the inflow and outflow are rather small compared to $u$; less than $1 \%$ of the maximum of $u$ but extend over a very large region in the $y-z$ plane. There is even a small, but appreciable, inflow at the top of the boundary layer. It should be noted that the cross-stream flow is rather weak and the cross-flow Reynolds number (see Saric ${ }^{2}$ ) is very small. As can be seen from this figure, the boundary layer thickness of the cross-flow $\left(\delta_{C}\right)$ is of the same size as the boundary layer thickness $(\delta)$ of the streamwise flow. In contrast, the magnitude of the cross-flow velocity component $\left(U_{c}\right)$ is very small compared to that of the mean flow $\left(U_{0}\right)$. It is clear that the cross-flow Reynolds number can be calculated by $R_{c}=\left(\delta_{c} / \delta\right)\left(U_{c} / U_{0}\right) R$. This gives a value $O(0.1)$.

Contours of the streamwise $(x)$ component of the vorticity, $\omega_{x}$, are plotted in Fig. 8 . These were obtained by numerically differentiating $v$ and $w$ using a second-order scheme. No smoothing of the results was done. It might have been expected that the numerical differentiation would induce substantial "noise," but none is apparent in the results shown in Fig. 8. The maximum and minimum of $\omega_{x}$ are $\pm 1.64 \times 10^{-4}$ and lie at $y=0.70$ and $z=\mp 3.95$. Positive $\omega_{x}$ indicates clockwise rotation and negative counterclockwise. It is seen from the structures shown in this figure that the bump generates a pair of counter-rotating vortices just above and on either side of it. These pump fluid down toward the

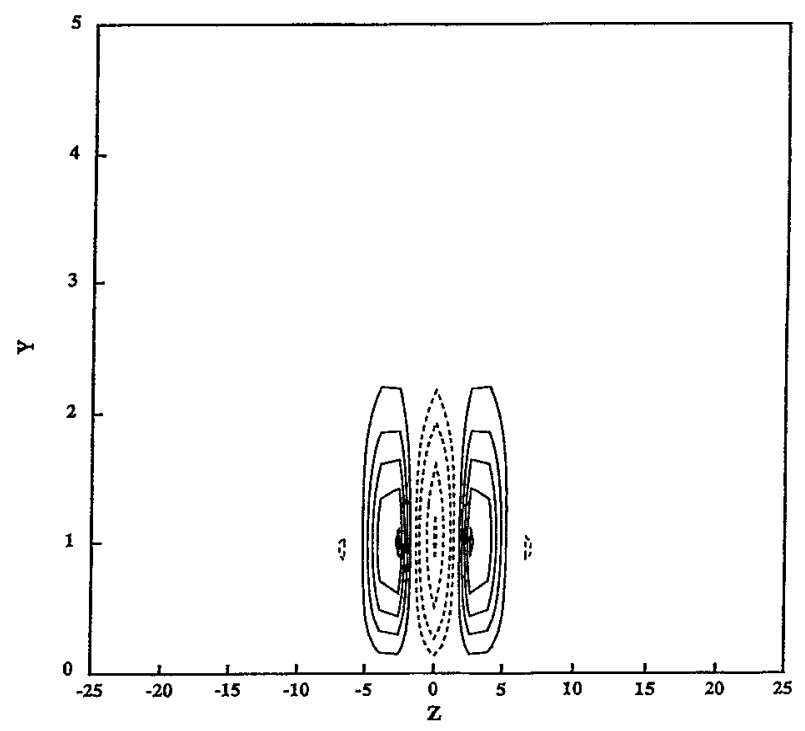

FIG. 9. Contours of $u$, the streamwise component of the disturbance velocity, on the $y-z$ plane at $x=709$. Contours with positive values of $u$ are solid and those with negative values are dashed. The contours values are $-1.6 \times 10^{-3}$ to $-0.2 \times 10^{-3}$ in steps of $0.2 \times 10^{-3}$ and from $-0.1 \times 10^{-3}$ to $0.5 \times 10^{-3}$ in steps of $0.1 \times 10^{-3}$, excluding 0.0 . The minimum value of $u$, $-0.63 \times 10^{-3}$, occurs on the centerline at $y=1.06$, with the maxima, $0.51 \times 10^{-3}$ being located at $y=1.06$ and $z= \pm 2.63$.

wall and into the upstream flowing "jet" of the disturbance field. Just above the main pair of vortices and slightly toward the centerline there are a weaker pair of oppositely rotating vortices. Between the main pair of vortices and the wall there is region of high vorticity due to the relatively strong outflow in the $\pm z$ directions.

This basic structure of the flow field persists farther downstream but is considerably weaker. This can be seen from the results shown in Figs. 9, 10, and 11. These results are on the $y-z$ planc at $x=709$, which is slightly downstream

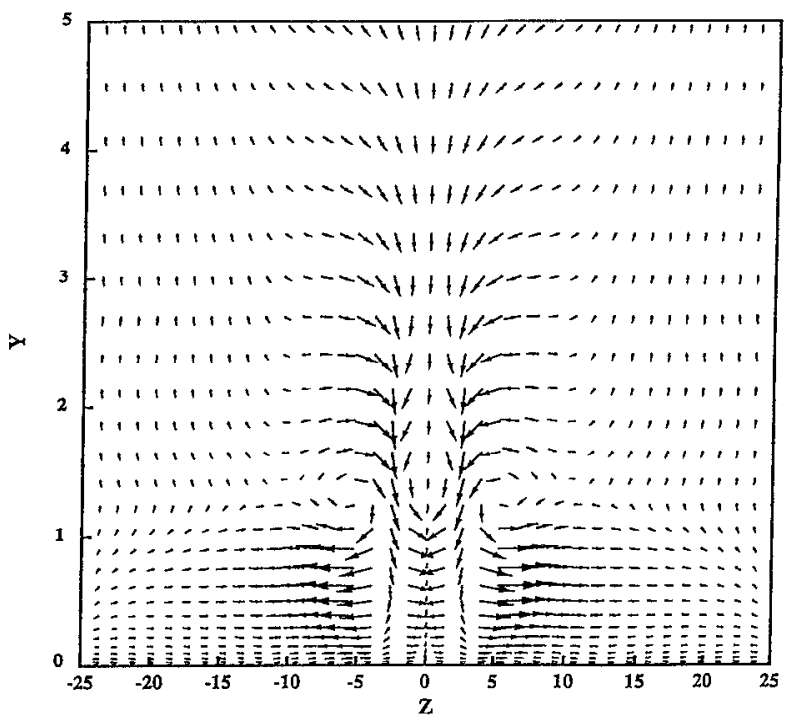

FIG. 10. Vectors of $(v, w)$ on the $y-z$ plane at $x=709$. The maxima of $\sqrt{v^{2}+w^{2}}$ are located at $y=0.62$ and $z= \pm 5.26$ and have the value $0.38 \times 10^{-5}$. 


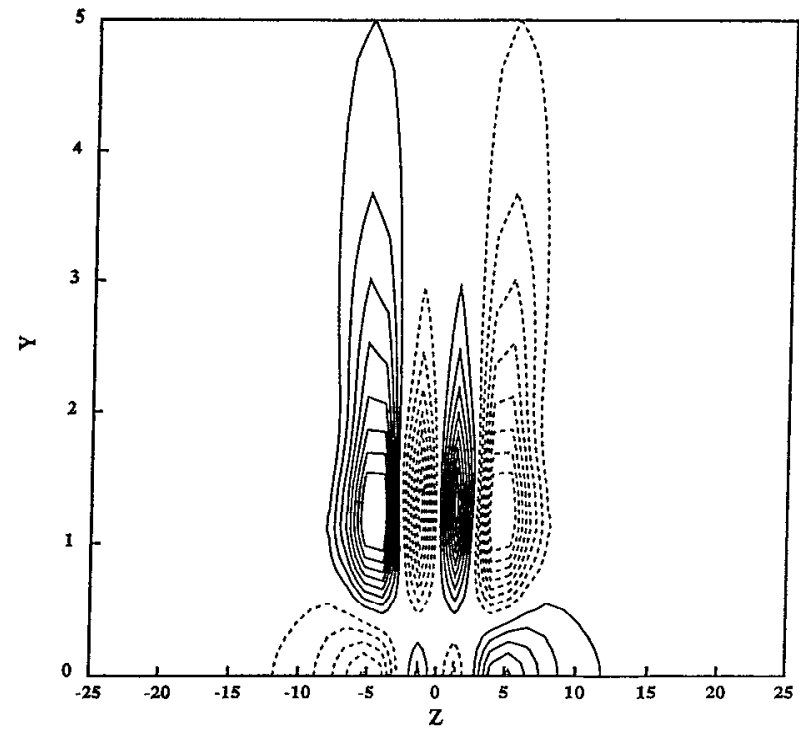

FIG. 11. Contours of $\omega_{x}$, the streamwise component of the disturbance vorticity, on the $y-z$ plane at $x=709$. Contours with positive values of $\omega_{x}$ are solid and those with negative values are dashed. Positive $\omega_{x}$ indicates clockwise rotation and negative counterclockwise. The contours values are $-1.6 \times 10^{-5}$ to $1.6 \times 10^{-5}$ in steps of $0.2 \times 10^{-5}$, excluding 0.0 . Note that these contour levels are one-tenth those of the contour plot of $\omega_{x}$ at $x=503$. The minimum value of $\omega_{x},-1.87 \times 10^{-5}$, is at $y=1.20$, and $z=3.95$, and the maximum, $1.87 \times 10^{-5}$, is at $y=1.20$ and $z=-3.95$.

of the section c-c, as shown in Fig. 1 of Gaster et al. ${ }^{1}$ and of this paper. At this location $R_{\delta^{*}} \approx 1585$. The contours of $u$ are shown in Fig. 9 with the same contour levels as in Fig. 6. The disturbance $u$ has the same general structure as at $x=503$. However, it is considerably weakened with the minimum of the upstream flowing jet only $-0.63 \times 10^{-3}$. The maxima of the downstream jets are, however, slightly larger than at $x=503$. The $y$ ' position of the center of these "jets" is 1.06 at this location as compared to 0.73 at $x=503$, and the "jets" have diffused in the wall normal direction. The $(v, w)$ vectors plotted in Fig. 10 also show uplift and spreading in $y$, as well as a general weakening. The maximum of $\sqrt{v^{2}+w^{2}}$ at this $x$ location is $0.38 \times 10^{-5}$, nearly seven times smaller than at $x=503$. The $z$ location of these maxima is exactly the same as at $x=503$, but the $y$ location is 0.62 as compared to $y=0.35$ at $x=503$. Finally, Fig. 11 shows contours of $\omega_{x}$ at $x=709$. The contour levels in this figure are one-tenth of those in Fig. 8. The general decrease and diffusive spreading in the vorticity is readily apparent. The main vortices have lifted farther from the wall. Their centers are at the same $z$ position as at $x=503$ but the $y$ position is now 1.20 instead of 0.70 and are at the same height as the secondary pair. The vorticity at the wall is also considerably weakened.

\section{CONCLUDING REMARKS}

The spatial evolution of the disturbance velocity field initiated from a shallow bump on a wall in a laminar boundary layer was computed by direct numerical simulation of the incompressible Navier-Stokes equations. Comparison of results from coarse- and fine-grid simulations showed that the coarse-grid simulation had converged. The evolution pattern and modal growth and decay trends were shown to be consistent with the experimental results of Gaster et al. ${ }^{1}$

The three-dimensional structure of the flow field was inferred by examining the velocity component and the streamwise component of the vorticity on two $y-z$ planes downstream of the bump. It was seen that the bump generates, at least in the farfield, a pair of counter-rotating vortices just above the wall and on either side of the bump location. These pump fluid down toward the wall and into an upstream flowing jet of the disturbance field. Outside of this main jet there are a pair of weaker downstream flowing jets. Just above the main pair of vortices and slightly toward the centerline there are a weaker pair of oppositely rotating vortices. Between the main pair of vortices and the wall there is region of vorticity due to the outflow in the $\pm z$ directions. As this flow evolves downstream, the vortices lift from the wall, diffuse, and weaken while maintaining their basic structure. The jets also weaken, diffuse, and lift, as must occur because they are, in a sense, both cause and effect of the vortices.

A theoretical study should be conducted to complete the understanding of this proposed linear transfer of energy between various spanwise modes. In addition, wind-tunnel and computational experiments should be conducted in order to understand the interaction of Tollmien-Schlichting waves with the bump-induced vorticity field.

\section{ACKNOWLEDGMENTS}

C.E.G. was supported, in part, by the National Aeronautics and Space Administration under NASA Contract No. NAS 1-19480 while in residence at the Institute for Computer Applications in Science and Engineering, NASA Langley Rescarch Center, Hampton, VA 23681-0001.

${ }^{1}$ M. Gaster, C. E. Grosch, and T. L. Jackson, "The velocity field created by a shallow bump in a boundary layer," Phys. Fluids 6, 3079 (1994).

${ }^{2}$ W. S. Saric, "Low-speed boundary-layer transition experiments," in Transition: Experiments, Theory \& Computations, edited by T. C. Corke, G. Erlbacher, and M. Y. Hussaini (Oxford University Press, Oxford, 1994).

${ }^{3}$ R. D. Joslin, C. L. Streett, and C.-L. Chang, "Validation of threedimensional incompressible spatial direct numerical simulation code-A comparison with linear stability and parabolic stability equations theories for boundary-layer transition on a flat plate," NASA TP-3205, 1992.

${ }^{4}$ R. D. Joslin, C. L. Streett, and C.-L. Chang, "Spatial direct numerical simulation of boundary-layer transition mechanisms: Validation of PSE theory," Theor. Comput. Fluid Dyn. 4, 271 (1993).

5 J. H. Williamson, "Low-storage Runge-Kutta schemes," J. Comput. Phys. 35, 48 (1980).

${ }^{6} \mathrm{C}$. L. Streett and M. Y. Hussaini, "A numerical simulation of the appearance of chaos in finite-length Taylor-Couette flow," Appl. Num. Math. 7, 41 (1991).

${ }^{7}$ G. Danabasoglu, S. Biringen, and C. L. Streett, "Spatial simulation of instability control by periodic suction and blowing," Phys. Fluids 3, 2138 (1991).

${ }^{8}$ C. L. Streett and M. G. Macaraeg, "Spectral multi-domain for large-scale fluid dynamic simulations," Int. J. Appl. Num. Math. 6, 123 (1989).

${ }^{9}$ R. D. Joslin and C. L. Streett, "The role of stationary crossflow vortices in boundary-layer transition on swcpt wings," Phys. Fluids 6, 3442 (1994). 